\title{
Notable advances
}

\section{Scores of papers were published this year, many of them reporting significant, if small, advances. Here are the papers we think had the biggest impact.}

The tumor suppressor p53 was thought to protect from cancer by monitoring cells

for DNA damage and eliminating potentially precancerous cells. But DNA damage may be irrelevant to $\mathrm{p} 53$ 's protective effects, researchers reported in September. Instead a second tumor suppressor, p19ARF, may be the crucial signal for p53 to kill precancerous cells. (Nature 443, 214-217)

$$
\text { 要 }
$$

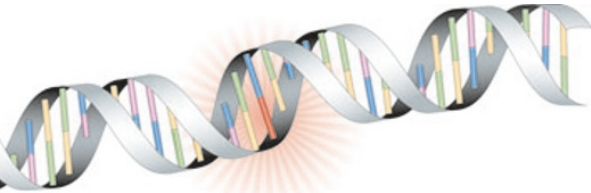

Three independent groups this year showed that HIV exhausts the ability of the immune system to combat infection by increasing the expression of a signaling molecule on $\mathrm{CD}^{+} \mathrm{T}$ cells and hampering their ability to respond to infected cells. The findings suggest a way to kick-start the immune system in HIV-infected individuals. (Nat. Med.12, 1198-1202; Nature 443, 350-354; J. Exp. Med. 203, 2281-2892)

The link between feeding and the brain's sensing of nutrient levels was further cemented this year when scientists showed that injecting leucine into mouse brains activates $\mathrm{mTOR}$, an enzyme known to regulate cell signaling and reduce eating. Rapamycin, an mTOR inhibitor, blocks this effect. The results suggest a possible target for obesity drugs. (Science 312, 927-930)

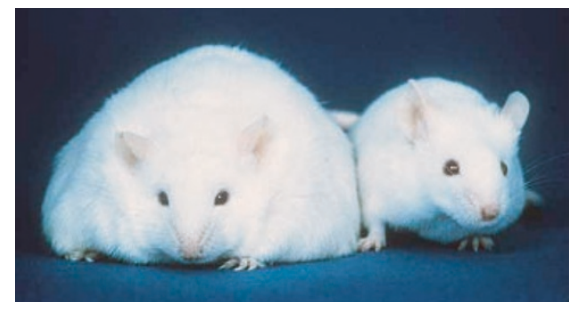

Treating cancer by targeting the stem cells that give rise to tumors runs the risk of also killing normal stem cells, such as those that generate blood. Two groups this year identified key differences between normal and leukemia-initiating stem cells that might help target only cancer stem cells while sparing their normal counterparts. (Nature 441, 475-483; Nature 441, 518-522)

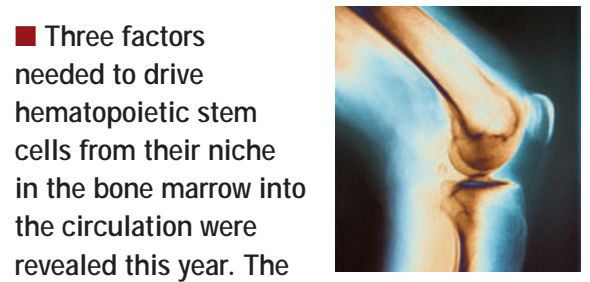
stem cells' mobilization requires sympathetic nervous system activity, a calcium-sensing receptor on their cell surface to tether the cells to the bone niche and the differentiation of osteoclasts, which releases enzymes to cleave tethering proteins. (Cell 124, 407-421; Nature 439, 599-603; Nat. Med. 12, 657-664)

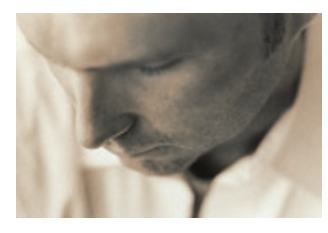

Antidepressants may take several weeks to act, and their effects can extend for months after their use has been discontinued. How? Depression in mice induces remodeling of nuclear chromatin, altering gene expression, researchers reported in April. These epigenetic changes are reversed by antidepressants and may explain the drugs' long-lasting effects. (Nat. Neurosci. 9, 519-525)

A new class of $\mathrm{CD4}^{+} \mathrm{T}$ cells, called $\mathrm{T}_{\mathrm{H}} 17$ cells, is thought to promote autoimmune disease. Two reports this year revealed that these cells are surprisingly closely linked to the protective regulatory $\mathrm{T}$ cells that dampen harmful immune responses: the cytokine TGF- $\beta$ drives the development of both types. (Nature 441, 235-238; Nature 441, 231-234)

\section{Activity of} the $\gamma$-secretase enzyme is crucial in Alzheimer disease. Researchers showed in September that presenilins, key components of the

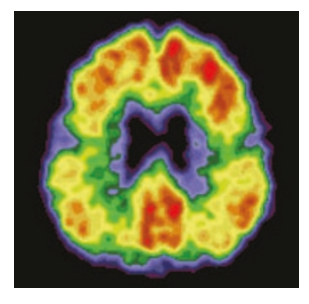
$\gamma$-secretase complex, form calcium channels in the endoplasmic reticulum. This channel function is independent of $\gamma$-secretase activity and is absent in mutant presenilins linked to genetic forms of Alzheimer disease, challenging the prevailing amyloid hypothesis. (Cell 126, 981-993)

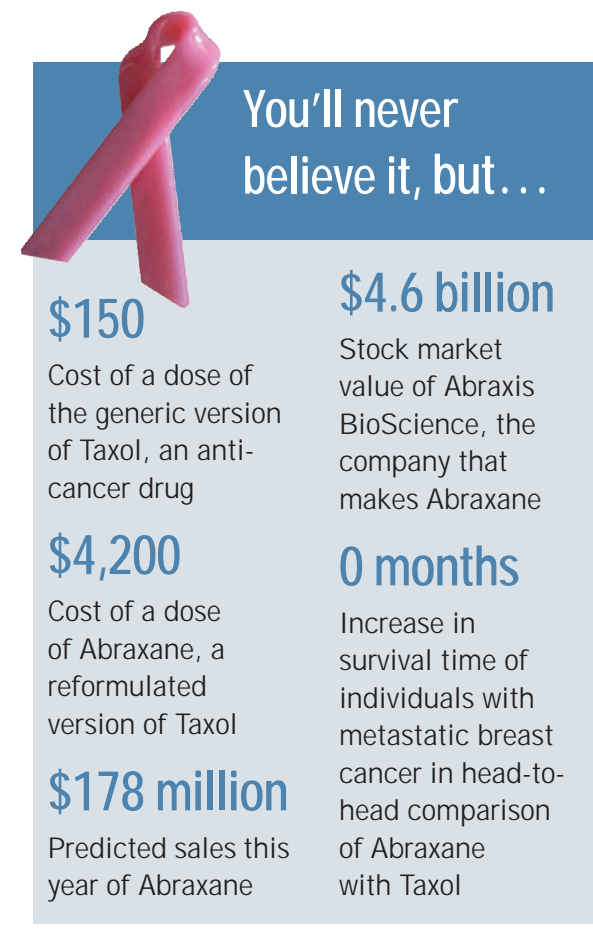

Two groups this year gave paralyzed individuals hope with remarkable advances in the area of brain-computer interfaces- or neuromotor prostheses. The interfaces enable a device implanted in the brain to translate electrical signals from neurons into external movement, allowing a tetraplegic man to move an electronic cursor as well as a robotic arm by thought. A second group increased the response speed of such interfaces. (Nature 442, 164-171;

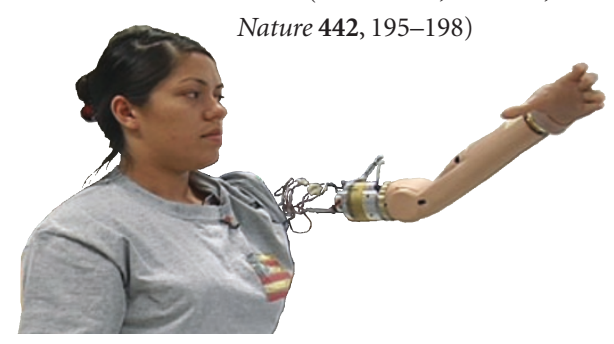

Two studies this year showed how the activity of TGF- $\beta$ cytokines contributes to vascular disease. The protein emilin prevents high blood pressure in mice caused by too much TGF- $\beta$ activity. Higher levels of another antagonist, soluble endoglin, are present in pregnant women who develop pre-eclampsiaa major cause of maternal and fetal deathand trigger pre-eclamptic symptoms in mice. (Cell 124, 929-942;

Nat. Med.12, 642-649; N. Engl. J. Med. 355, 992-1005) 